COMPUTATIONAL METHODS IN APPLIED MATHEMATICS, Vol. 8 (2008), No. 1, pp. 3-20

(C) 2008 Institute of Mathematics of the National Academy of Sciences of Belarus

\title{
ANALYSIS OF UPWIND METHOD FOR PIECEWISE DETERMINISTIC MARKOV PROCESSES
}

\author{
M. ANNUNZIATO ${ }^{1}$
}

\begin{abstract}
In this paper we investigate the behaviour of the numerical solution of the Liouville - Master Equation (LME) for time dependent distributions, that arises by the statistical characterisation of a class of piecewise deterministic stochastic processes (PDP). This is a system of linear hyperbolic PDEs with non-constant coefficients. The numerical solution is found by means of upwind and forward Euler scheme. We find a Courant - Friedrichs - Lewy condition ensuring both convergence and monotonicity of the numerical solution. In particular, the global error is shown to be bounded by a linear increasing in the integration time, under an appropriate norm. Some numerical tests for known analytical solutions of practical problems verify the theoretical findings.
\end{abstract}

2000 Mathematics Subject Classification: 65M06, 65M12, 65M15, 60K40, 60J75.

Keywords: upwind, piecewise-deterministic process, random telegraph process, binary noise, dichotomic noise, non-conservative system, balance law.

\section{Introduction}

We deal with the following system of first order hyperbolic partial differential equations:

$$
\partial_{t} F_{l}(x, t)+A_{l}(x) \partial_{x} F_{l}(x, t)=\sum_{s=1}^{S} Q_{l s} F_{s}(x, t)
$$

for the unknown functions $F_{l}(x, t)$, with the Cauchy initial conditions:

$$
F_{l}(x, 0)=F_{0 l}(x)=\int_{-\infty}^{x} p_{0 l}\left(x^{\prime}\right) d x^{\prime}
$$

given for each $l=\{1, \ldots, S\}$ of the system, and $\sum_{l=1}^{S} Q_{l s}=0$.

Equation (1.1) represents the Liouville-Master Equation for a piecewise deterministic process (PDP) with Poisson statistics of the transition events. PDPs were introduced by Davis [8] as a wide class of stochastic processes that involves deterministic motion punctuated by random switches of the motion. Here we study a restricted case on the line of Ref. [22], i. e. a first order differential equation driven by the Markov process. For PDPs that are associated with Eq. (1.1), we give the following:

${ }^{1}$ Dipartimento di Matematica e Informatica, Università degli Studi di Salerno, Via Ponte Don Melillo 84084 Fisciano (SA). Italy E-mail: mannunzi@unisa.it 
Definition 1.1. We say that $X(t):\left[t_{0}, \infty\right] \rightarrow \Omega$ is a continuous PDP if:

(a) $X(t)$ is the solution of:

$$
\frac{d X(t)}{d t}=A_{l}(X)
$$

where $A_{l}: \Omega \rightarrow \mathbb{R}$ is a function chosen randomly from a set of $\left\{A_{1}, \ldots, A_{S}\right\}$ known functions. Given $A_{l}$, we say that the dynamics is in the state $l$. We require that $A_{l}(x)$ be Lipschitz continuous, so that for fixed $l, X(t)$ exists, is unique and non-explosive solution.

(b) The initial condition is settled by the Cauchy problem to Eq. (1.3), i.e. $X\left(t_{0}\right)=X_{0}$, and by the initial state of the process.

(c) Each state is characterised by a Poisson statistics of the switching times:

$$
\mu_{l} e^{-\mu_{l} \tau}
$$

where $\tau$ is the time between two switches, and $\mu_{l} \in\left\{\mu_{1}, \ldots, \mu_{S}\right\}$.

$(d)$ If the process is in the state $j\left(A_{j}\right)$, when a switching time occurs, the state can switch to the state $i\left(A_{i}\right)$ according to the transition probability matrix (or stochastic matrix):

$$
\left\{q_{i j}\right\}
$$

having the following fundamental [14] properties: $0 \leqslant q_{i j} \leqslant 1$, for each $i, j \in 1, \ldots, S$, and $\sum_{i=1}^{S} q_{i j}=1$, for each $j \in 1, \ldots, S$.

Assumptions $(a),(c)$ and $(d)$ define the three local characteristics of the PDP [8]. We can easily visualise the process $X(t)$ as a sequence of solutions of Eq. (1.3), where the end point of each is just the initial condition for the next one. Hence this process is continuous, because at switching times only the first derivative of $X(t)$ is involved. Assumption (c) makes the process be Markovian, since the exponential statistics, regarded as generated by an infinitesimal time process, corresponds to a constant switching rate, namely it has no memory $^{1}$ of the time past since the last switch. The unknown functions $F_{l}(x, t)$ for Eq. (1.1) represent the distribution functions associated to the process $X(t): F_{l}(x, t):=\mathbb{P}\{X(t) \leqslant$ $x$, state $=l\}$, i.e. the probability of finding the process under the value $x$ and in the state $l$. $\mathbb{P}$ is a probability measure of an abstract probability space that for our pourposes does not need to be specified. The matrix $Q_{l s}$ is related to $q_{l s}$ by: $Q_{l s}=\left(q_{l s}-\delta_{l s}\right) \mu_{s}$, where $\delta_{l s}$ is the Kronecker's symbol.

Eq. (1.1) is written for $F_{l}(x, t)$ rather than the densities $p_{l}(x, t)$, but, for an absolute continuous measure, it is: $F_{l}(x, t)=\int_{-\infty}^{x} p_{l}\left(x^{\prime}, t\right) d x^{\prime}$. We remind that $p_{l}(x, t) \geqslant 0$, so that $F_{l}(x, t)$ is non-decreasing in $x$.

Our aim is to find solutions $F_{1}(x, t), \cdots, F_{S}(x, t)$ of Eq. (1.1), starting from Cauchy (1.2), and then the total distribution function:

$$
\mathcal{F}(x, t):=\sum_{l=1}^{S} F_{l}(x, t),
$$

representing the distribution of the process at time $t$, regardless of the states of it. We note that the conservation of probability measure requires that the conditions $\lim _{x \rightarrow \infty} \mathcal{F}(x, t)=1$ and $\lim _{x \rightarrow-\infty} \mathcal{F}(x, t)=0$ have to be satisfied.

The general class of PDP has application in operation research, but, although not presented in such that formulation, we found also application to science and engineering in:

\footnotetext{
${ }^{1}$ See Ref. [2] for PDPs with memory.
} 
anomalous diffusion [3], reaction-diffusion [15], scattering of radiation [17], biological dispersal $[11,26]$, non-Maxwellian equilibrium $[1,5,13,20,33]$, diagnostic technique for semiconductor lasers [18], filtered telegraph signal [18,27], harmonic oscillators [21]. The statistical properties for processes like these, can be described by using, for example, the characteristic functional method [4,5], the Monte Carlo simulation, various spectral representations (Fourier, Laplace, generating functions, characteristic function [18, 23]), direct probabilistic methods $[10,11]$, or the associated probability density distributions $p_{1,2}(x, t)$ that are governed by a Chapman - Kolmogorov equation.

For example, let $X(t)$ be a dissipative process subject to a noised input $\xi(t)$, described by the equation:

$$
\frac{d X}{d t}=-\gamma X(t)+W \xi(t)
$$

Here $\xi(t)$ represents a dichotomic noise (or random telegraph signal [17,27]), that takes on values \pm 1 , with Poisson statistics $\mu e^{-\mu \tau}$. The solution of Eq. (1.7) is composed of pieces of increasing and decreasing exponentials (i.e. deterministic functions), but the whole process $X(t)$ is not deterministic, it represents a random sample path in a probability space. The density probability distribution satisfies a Liouville - Master Equation ${ }^{1}$ of the following form $[1,18,35]$ :

$$
\left\{\begin{array}{l}
\partial_{t} p_{1}-(x-1) \partial_{x} p_{1}=(1-\mu) p_{1}+\mu p_{2} \\
\partial_{t} p_{2}-(x+1) \partial_{x} p_{2}=\mu p_{1}+(1-\mu) p_{2}
\end{array}\right.
$$

when $\gamma=W=1$.

We note that if in Eq. (1.3) and (1.1), $A_{1}(x)=-x+1, A_{2}(x)=-x-1$, and $\mu_{1}=\mu_{2}=\mu$, $q_{l s}=1$ for $l \neq s$ and $q_{l s}=0$ for $l=s$, then Eq. (1.7) and its associated Liouville-Master Equation (1.8) are recovered. This shows an alternative way of thinking a dynamics subject to dichotomous noise, as a case of piecewise deterministic process.

In literature, most of the numerical treatment for hyperbolic systems is developed for conservation laws of the form: $u_{t}+f(u)_{x}=0$. We mention upwind, Godunov, TVD and WENO as examples of well known schemes that preserve conservation and monotonicity of the solution $[16,30,34]$. However, the way of how to discretize the transport operator is still an open issue [31]. Sometimes a coupling or source term between the conservation equations is included $[6,19]$, then classical numerical schemes can be applied after some appropriate modifications, because important features, such as stability and monotonicity (e.g., see [6]), can be destroyed by the presence of these terms. On the other hand, some transport phenomena can be described by a non-conservative hyperbolic system, e.g. the advection with non-constant velocity field: $u_{t}+a(x, t) u_{x}=0$ or the quasi-linear system $u_{t}+f(u) u_{x}=0$, for which extensions of conservative schemes can be performed, as shown in the recent paper [25].

The present equations of system (1.1) are written in non-conservative (advective) form with a linear coupling (balance) between the unknown $F_{s}(x, t)$. The solutions of system (1.1) are positive and monotonically non-decreasing, and it is essential that the same properties be valid for the approximated solutions. Here we use a first order upwind method with an explicit Euler scheme for the time stepping, i.e., the Courant - Isaacson - Rees

\footnotetext{
${ }^{1}$ In general, equations for density probability of random processes are derived from the Chapman Kolmogorov equation. As discussed in Ref. [14] the same equation becomes a Liouville equation, in absence of randomness, and a Master Equation, if only jump processes are involved. We use both terms in order to stress the deterministic and the random character of the processes considered here.
} 
scheme. This is well known and quite robust for advection, but we want to investigate the influence of the coupling on the numerical solution. Monotonicity is guaranteed, while a CFL-like condition ensure stability. The estimate of the global error that is norm dependent is also important, and its appropriate choice of it can refine the estimate. Thanks to some equilibrium solutions for dichotomous processes, known in analytical closed form, we can validate the theoretical results, also fot less regular solutions. Moreover, we have found the expression of the equilibrium solution for a relaxation process with many states, as Legendre polynomials series, useful for validating the findings for systems larger than two. Finally, we note that from the results of this paper we can know the distribution functions out of equilibrium for this important class of processes, which is a rare case.

In the next section we give some preliminaries concerning the range of study of the solutions and establish the discretization technique used. Sections 3 and 4 are devoted to the investigation of the convergence and monotonicity of the numerical solution of Eq. (1.1). In Sections 5 we give the test results for convergence of the numerical solution as applied to practical models. Appendix gives the equilibrium conditions for the process and the analytical equilibrium solution for a relaxation process with many states.

\section{Preliminaries}

2.1. Preliminary considerations on the equilibrium solution. This system of equations is linear hyperbolic, with non-constant coefficients. We will focus our attention on solutions $\mathcal{F}(x, t)$ having an equilibrium on a finite segment $\Omega=[a, b]$ of the real axis. Conditions for existence of the equilibrium solution can be conjectured by using simple dynamical considerations [23]. If all dynamical equations (1.3) own only attraction points and all of these are contained at the intersection of the basin of attraction of each, then a process starting from this region will never escape. Whence, there should exist a closed subset $\Omega$ of the real axis where the process is confined and a stationary distribution $\mathcal{F}_{\text {eq }}(x)=$ $\lim _{t \rightarrow \infty} \mathcal{F}(x, t)$ exists. Appendix A gives the condition for the functions $A_{l}(x)$ related to this requirement.

2.2. The discrete equation. Due to its hyperbolic nature, we apply the upwind scheme jointly with forward Euler time stepping $[16,24,30]$ to numerically solve Eq. (1.1). In the domain $(\Omega \times[0, T])$ we introduce a uniform mesh:

$$
\left(x_{k}, t_{i}\right)\left\{\begin{array}{l}
k=0, \ldots, N_{k}, \\
i=0, \ldots, N, \quad N=T / \Delta t
\end{array}\right.
$$

with steps $\Delta x=x_{k+1}-x_{k}$ and $\Delta t=t_{n+1}-t_{n}$, so that the functions $A_{l}(x)$ are written as ${ }^{l} A_{k}=A_{l}\left(x_{k}\right)$, and the discrete solution as ${ }^{l} F_{k}^{i} \approx F_{l}\left(x_{k}, t_{i}\right)$. The upwind scheme takes the discrete derivative $x$-direction according to the sign of $A_{l}(x)$, so that we obtain the following finite difference scheme for ${ }^{l} F_{k}^{i}$ :

$$
{ }^{l} F_{k}^{i+1}={ }^{l} F_{k}^{i}+\Delta t\left(-{ }^{l} A_{k} \frac{{ }^{l} F_{k+\nu}^{i}-{ }^{l} F_{k+\nu-1}^{i}}{\Delta x}+\sum_{s} Q_{l s}{ }^{s} F_{k}^{i}\right),
$$

where

$$
\nu=\left\{\begin{array}{lll}
1, & \text { if } & { }^{l} A_{k}<0 \\
0, & \text { if } & { }^{l} A_{k}>0
\end{array}\right.
$$


This is a first order explicit scheme, where the solutions ${ }^{l} F_{k}^{n}$, at the time step $n$, are calculated starting from the Cauchy initial conditions ${ }^{l} F_{k}^{0}=F_{0 l}\left(x_{k}\right)$. The total distribution function, approximating (1.6), is:

$$
\mathcal{F}_{k}^{i}:=\sum_{l}{ }^{l} F_{k}^{i}
$$

The conditions for the convergence of Eq. (2.2) can be obtained by following a classical analysis, i.e. requiring that the time step advancing operator, of the numerical scheme, be not much greater than $1[16,24,30]$, so that the global error can be bounded by some constant. If $Q=0$, it is known that the numerical scheme (2.2) is stable when $(\max |A| \Delta t)<\Delta x$ is fulfilled (CFL). If $Q \neq 0$, for a generic operator, the global error is bounded by an exponential increase in the time. However this estimate depends on the norm, that can be refined by an opportune choice. All these facts are well known for a scalar PDE [16], and have to be extended to our system.

Equation (2.2) can be rewritten as a matrix by vector product: $\mathbf{v}^{i+1}=B \mathbf{v}^{i}$, where $\mathbf{v}^{i}=\left(\cdots{ }^{1} F_{k}^{i} \cdots, \cdots{ }^{2} F_{k}^{i} \cdots, \ldots, \cdots{ }^{S} F_{k}^{i} \cdots\right)^{\top}$ is the vector of numerical solutions ${ }^{l} F_{k}^{i}$, having queued the state components to a single vector, and $B$ is the advancing operator, i.e. a $S N_{x} \times S N_{x}$ matrix. This matrix reads as: $B=(I-(\Delta t / \Delta x) U+\Delta t \tilde{Q})$. $\tilde{Q}$ is a block matrix, where each block is diagonal $Q_{l s} I$ of order $N_{x}$, and $U$ is block diagonal, with blocks $U$ of tri-diagonal form due to upwinding:

$$
{ }^{l} U_{k, k}=-\left|{ }^{l} A_{k}\right|, \quad{ }^{l} U_{k, k+1}=\max \left(-{ }^{l} A_{k}, 0\right), \quad{ }^{l} U_{k, k-1}=\max \left({ }^{l} A_{k}, 0\right) .
$$

Usually we have $\|B\|<1+h$ with $h>0$, for 1 and $\infty$ norms, so that from this analysis, the global error is bounded only by an exponential increase in the time $e^{h N}$. This is enough to ensure convergence for a finite time interval of integration, nevertheless a better estimate of the global error would be valuable, because, as mentioned in the former subsection, we are interested in finding a stationary solution that could be reached after long time. For this reason we calculate the errors as in the next section.

2.3. Global errors and convergence. The global pointwise error is defined as the difference between the exact and the approximated solution:

$$
{ }^{l} e_{k}^{i}:=F_{l}\left(x_{k}, t_{i}\right)-{ }^{l} F_{k}^{i} .
$$

In order to establish convergence, we define the following norms:

$$
\begin{gathered}
{ }^{l} E^{i}:=\left\|{ }^{l} e^{i}\right\|_{\infty}=\max _{k}\left|{ }^{l} e_{k}^{i}\right|, \\
\left\|E^{i}\right\|_{1}:=\left\|e^{i}\right\|_{\infty, 1}=\sum_{l=1}^{S}{ }^{l} E^{i} .
\end{gathered}
$$

The $\infty$-norm is used for the space index, while the 1-norm is a natural choice for the discrete states.

We define the global pointwise error, for the total distribution, as:

$$
\hat{E}^{i}:=\max _{k}\left|\mathcal{F}\left(x_{k}, t_{i}\right)-\mathcal{F}_{k}^{i}\right|,
$$

which is related to (2.6) by

$$
\hat{E}^{i} \leqslant \max _{k} \sum_{l}\left|{ }^{l} F\left(x_{k}, t_{i}\right)-{ }^{l} F_{k}^{i}\right| \leqslant\left\|E^{i}\right\|_{1} .
$$


2.4. Local truncation error. The local truncation error ${ }^{l} \mathcal{E}_{k}^{i}$ is defined, as usual, by substituting the exact solution $F_{l}\left(x_{k}, t_{i}\right)$ at mesh grid points into the numerical scheme $(2.2)$

$$
\begin{gathered}
l_{\mathcal{E}_{k}^{i}}^{i}:=\frac{F_{l}\left(x_{k}, t_{i}+\Delta t\right)-F_{l}\left(x_{k}, t_{i}\right)}{\Delta t}+ \\
A_{l}\left(x_{k}\right)\left(\frac{F_{l}\left(x_{k}+\nu \Delta x, t_{i}\right)-F_{l}\left(x_{k}+(\nu-1) \Delta x, t_{i}\right)}{\Delta x}\right)-\sum_{s} Q_{l s} F_{s}\left(x_{k}, t_{i}\right),
\end{gathered}
$$

and evaluating it by using the Taylor expansion

$$
{ }^{l} \mathcal{E}_{k}^{i}=\frac{\Delta x}{2}\left(\alpha \partial_{t}^{2} F_{l}\left(x_{k}, \xi_{k}\right)-\left|A_{l}\left(x_{k}\right)\right| \partial_{x}^{2} F_{l}\left(\eta_{i}, t_{i}\right)\right),
$$

where $\alpha:=\Delta t / \Delta x$, and $\xi_{k}$ and $\eta_{i}$ are unknown points. As for the global error, we use the $\infty$-norm for the spatial variable:

$$
{ }^{l} \mathcal{E}^{i}:=\left\|{ }^{l} \mathcal{E}^{i}\right\|_{\infty}=\max _{k}\left|{ }^{l} \mathcal{E}_{k}^{i}\right|
$$

and the discrete $L^{1}$ norm for the states

$$
\left\|\mathcal{E}^{i}\right\|_{1}:=\left\|\mathcal{E}^{i}\right\|_{\infty, 1}=\sum_{l=1}^{S}{ }^{l^{C}}{ }^{i}
$$

\section{Convergence analysis}

Here we prove convergence of ${ }^{l} F_{k}^{n}$ to $F_{l}\left(x_{k}, t_{n}\right)$ starting from the global error with the norm defined by (2.7). It shows a linear rather than exponential increase in the time. This remarkable property is a direct consequence of the stochastic transition matrix (1.5).

Theorem 3.1. Let $F_{l}(x, t) \in C^{2,2}(\Omega \times[0, T])$ be a solution of Eq. (1.1) with $\left|A_{l}(x)\right| \leqslant M$ and the Cauchy conditions (1.2) for $x \in \Omega$ and $l \in\{1, \ldots, S\}$, where $\Omega$ is a closed set of the real axis. Fix the uniform mesh $(2.1)$ on $(\Omega \times[0, T])$, of step-size $\Delta x$ and $\Delta t$, if:

$$
\Delta t<\left(M / \Delta x+\max _{l}\left|Q_{l l}\right|\right)^{-1},
$$

then the global error $\hat{E}^{n}(2.7)$, associated with the numerical solution $\mathcal{F}_{k}^{n}(2.3)$, resulting from the numerical scheme (2.2) and computed at the time step $n=T / \Delta t$, is bounded by $\left\|E^{n}\right\|_{1} \leqslant\left\|E^{0}\right\|_{1}+\mathcal{E} n \Delta t$. Provided that the maximum local truncation error $\mathcal{E}=\max _{i}\left\|\mathcal{E}^{i}\right\|_{1}$ is $\mathcal{O}(\Delta x)$ for any $(x, t) \in \Omega \times[0, T], \mathcal{F}_{k}^{n}$ converges to $F_{l}\left(x_{k}, t_{n}\right)$ as $\Delta x \rightarrow 0$ for $(x, t) \in \Omega \times[0, T]$.

Proof. We search for the convergence of the numerical method by studying the global pointwise error (2.4) associated to (2.2):

$$
{ }^{l} e_{k}^{i+1}={ }^{l} e_{k}^{i}+\Delta t\left(-{ }^{l} A_{k} \frac{{ }^{l} e_{k+\nu}^{i}-{ }^{l} e_{k+\nu-1}^{i}}{\Delta x}+\sum_{s} Q_{l s}{ }^{s} e_{k}^{i}\right)-{ }^{l} \mathcal{E}_{k}^{i} \Delta t,
$$

where ${ }^{l} \mathcal{E}_{k}^{i}$ is the local truncation error (2.9). Taking the modulus and using the triangle inequality gives

$$
\left|{ }^{l} e_{k}^{i+1}\right| \leqslant\left(|1-\alpha|{ }^{l} A_{k}\left|+\Delta t Q_{l l}\right|+\alpha\left|{ }^{l} A_{k}\right|\right){ }^{l} E^{i}+\Delta t \sum_{s \neq l} Q_{l s}{ }^{s} E^{i}+{ }^{l} \mathcal{E}^{i} \Delta t .
$$


By virtue of the condition (3.1), the first modulus on the right side is positive

$$
1-\alpha\left|{ }^{l} A_{k}\right|+\Delta t Q_{l l} \geqslant 0 \quad \forall l, k,
$$

then we get

$$
\left|{ }^{l} e_{k}^{i+1}\right| \leqslant\left(1+\Delta t Q_{l l}\right){ }^{l} E^{i}+\Delta t \sum_{s \neq l} Q_{l s}{ }^{s} E^{i}+{ }^{l} \mathcal{E}^{i} \Delta t .
$$

This inequality is valid for all mesh points $x_{k}$ and the r.h.s does not depend on $k$, then we can write

$$
{ }^{l} E^{i+1} \leqslant \sum_{s}(I+\Delta t Q)_{l s}{ }^{s} E^{i}+{ }^{l} \mathcal{E}^{i} \Delta t .
$$

Taking the sum over all states $l$, we obtain the inequality written for the discrete $L^{1}$ norm on the states

$$
\left\|E^{i+1}\right\|_{1} \leqslant\|(I+\Delta t Q)\|_{1}\left\|E^{i}\right\|_{1}+\left\|\mathcal{E}^{i}\right\|_{1} \Delta t .
$$

The matrix $I+\Delta t Q$ gives us information about the convergence of the numerical method (2.2). Since $(I+\Delta t Q)_{l l} \geqslant 0$, because of (3.4) and $Q_{l s}=q_{l s} \mu_{s} \geqslant 0$ for $l \neq s$, we have

$\left.\|(I+\Delta t Q)\|_{1}=\max _{s} \sum_{l} \mid \delta_{l s}+\Delta t\left(q_{l s}-\delta_{l s}\right) \mu_{s}\right) \mid=\max _{s}\left(\sum_{l} \delta_{l s}\left(1-\Delta t \mu_{s}\right)+\Delta t \mu_{s} \sum_{s} q_{l s}\right)=1$,

from the fundamental property (1.5) of the stochastic transition matrix $q_{l s}$. By iterating (3.8), the error of the numerical method at the time step $n$ is bounded by

$$
\left\|E^{n}\right\|_{1} \leqslant\left\|E^{0}\right\|_{1}+n \Delta t \mathcal{E}
$$

provided that the maximum local truncation error $\mathcal{E}=\max _{i}\left\|\mathcal{E}^{i}\right\|_{1}$ is $\mathcal{O}(\Delta x)$ for any $(x, t) \in$ $(\Omega \times[0, T])$.

3.1. Smearing. In Theorem 3.1 we have restricted our analysis to solutions having both a second spatial and a temporal derivative, but in general local discontinuities can be considered because the upwind method tends to regularise the solution. This is explained in terms of modified equation [30,31] modelling the numerical method. If in Eq. (1.1) we consider also the local truncation error (2.9), we get

$$
\partial_{t} w+A(x) \partial_{x} w-Q w+\frac{\Delta t}{2} \partial_{t}^{2} w-\frac{|A(x)| \Delta x}{2} \partial_{x}^{2} w \simeq 0
$$

having omitted the indexes. Taking the derivatives $\partial_{t}$ and $\partial_{x}$ of Eq. (3.9)

$$
\partial_{t}^{2} w+A(x) \partial_{t x} w-Q \partial_{t} w+\mathcal{O}\left(\Delta t^{2}\right)+\mathcal{O}\left(\Delta x^{2}\right)=0
$$

and

$$
\partial_{x t} w+A^{\prime}(x) \partial_{x} w+A(x) \partial_{x}^{2} w-Q \partial_{x} w+\mathcal{O}\left(\Delta t^{2}\right)+\mathcal{O}\left(\Delta x^{2}\right)=0 .
$$

Merging these two equations and cancelling $\partial_{x t}$

$$
\partial_{t}^{2} w=A(x)\left(A^{\prime}(x)-Q\right) \partial_{x} w+A^{2}(x) \partial_{x}^{2} w+Q \partial_{t} w+\mathcal{O}\left(\Delta t^{2}\right)+\mathcal{O}\left(\Delta x^{2}\right),
$$

then inserting it into Eq. (3.9), gives us

$$
\left(I+\frac{\alpha \Delta x}{2} Q\right) \partial_{t} w+A(x)\left[I+\frac{\alpha \Delta x}{2}\left(\partial_{x} A-Q\right)\right] \partial_{x} w-Q w=\frac{\Delta x}{2}\left(|A(x)|-\alpha A^{2}(x)\right) \partial_{x}^{2} w
$$


refered to as the modified equation. When $\Delta x \rightarrow 0$, Eq. (1.1) is recovered. If $\Delta x$ is small enough, the matrix coefficient in front of $\partial_{t}$ is almost an identity, and when the CFL (3.1) condition is satisfied, the coefficients of $\partial_{x} w$ are always non-negative, so that the solutions have a diffusive (viscous) character of a parabolic equations. The discrete scheme (2.2) appears to be a second order scheme with respect to Eq. (3.10), therefore the numerical solution ${ }^{l} F_{k}^{i}$ gives a better approximation to this modified equation than Eq. (1.1), which explains the viscous behavior.

\section{Nondecreasing property}

The solutions of Eq. (1.1) are nondecreasing functions in $x$. It is desirable that the numerical solutions ${ }^{l} F_{k}^{i}$ have the same property or equivalently, that the discrete density probability distributions defined as

$$
{ }^{l} p_{k}^{i}:=\left({ }^{l} F_{k}^{i}-{ }^{l} F_{k-1}^{i}\right) / \Delta x
$$

will never become negative for all $k$ and $i>0$.

Positivity conditions are well known for conservation laws, now we test them for system (2.2) in the presence of the operator $Q$. The proof will be given for semi-discrete system, since general positivity theorems are known [16].

Theorem 4.1 (Monotonicity). Let ${ }^{l} F_{k}^{i}$ be the numerical solution obtained with the use of the numerical scheme (2.2), and ${ }^{l} F_{k}^{0}-{ }^{l} F_{k-1}^{0} \geqslant 0$. If the CFL condition (3.1) is satisfied, then ${ }^{l} F_{k}^{i}-{ }^{l} F_{k-1}^{i} \geqslant 0 \forall i>0, \forall l, \forall k$.

Proof. By subtracting the solution at mesh points $k$ and $k+1$ we find:

$$
\begin{gathered}
{ }^{l} F_{k}^{i+1}-{ }^{l} F_{k-1}^{i+1}={ }^{l} F_{k}^{i}-{ }^{l} F_{k-1}^{i}+\Delta t\left(-\frac{{ }^{l} A_{k}}{\Delta x}{ }^{l} F_{k+\nu}^{i}+\frac{{ }^{l} A_{k}}{\Delta x}{ }^{l} F_{k+\nu-1}^{i}+\right. \\
\left.\frac{{ }^{l} A_{k-1}}{\Delta x}{ }^{l} F_{k+\nu-1}^{i}-\frac{{ }^{l} A_{k-1}}{\Delta x}{ }^{l} F_{k+\nu-2}^{i}+\sum_{s} Q_{l s}\left({ }^{s} F_{k}^{i}-{ }^{s} F_{k-1}^{i}\right)\right) .
\end{gathered}
$$

Let $\Delta x p_{k}^{i+1}={ }^{l} F_{k}^{i+1}-{ }^{l} F_{k-1}^{i+1}$, then

$$
{ }^{l} p_{k}^{i+1}={ }^{l} p_{k}^{i}+\Delta t\left(\frac{\left|{ }^{l} A_{k+\nu-1}\right|}{\Delta x} p_{k+2 \nu-1}^{i}+\sum_{s}\left(Q_{l s}-\frac{\left|{ }^{l} A_{k-\nu}\right|}{\Delta x} \delta_{l s}\right){ }^{s} p_{k}^{i}\right) .
$$

It is convenient to rewrite this equation as a semi-discrete scheme for $p_{k}(t)$ :

$$
\frac{d^{l} p_{k}(t)}{d t}=\frac{\left|{ }^{l} A_{k+\nu-1}\right|}{\Delta x} l_{p_{k+2 \nu-1}}(t)+\sum_{s}\left(Q_{l s}-\frac{\left|{ }^{l} A_{k-\nu}\right|}{\Delta x} \delta_{l s}\right){ }^{s} p_{k}(t)
$$

and observe that $Q_{l s} \geqslant 0$ when $l \neq s$, so that Eq. (4.4) is positive and ${ }^{l} p_{k}(t) \geqslant 0$. When time integration is performed by the forward Euler method, positivity is maintained by the condition [16]

$$
\max _{l, k}\left(\left|{ }^{l} A_{k-\nu}\right| / \Delta x-Q_{l l}\right) \Delta t \leqslant 1
$$

Remark 4.1. The CFL condition (3.1) is sufficient for both the convergence and the monotonicity (or nonnegativity of ${ }^{l} p_{k}^{i}$ ) of the numerical solution ${ }^{l} F_{k}^{i}$. 


\section{Numerical tests}

We have performed some numerical tests for Eq. (2.2) for a choice of $A_{l}(x)$ such that the analytical equilibrium solution $\mathcal{F}_{\text {eq }}(x)=\lim _{t \rightarrow \infty} \mathcal{F}(x, t)$ is known. Uniqueness can be guaranteed if the stochastic matrix $\left\{q_{i j}\right\}$ is irreducible. In this way we can test the numerical error (2.4) at least at equilibrium. We start from the Cauchy condition $H(x) / S$, where $H(x)$ is the Heavyside function (Riemann problem). Such that the choice corresponds to the puting of a particle at the origin in a randomly chosen state with a uniform distribution. Afterwards we integrate until equilibrium is reached. The discontinuous initial condition does not fulfils the hypothesis for convergence, but, as above mentioned, it is subject to smearing that tends to reguralize discontinuities (see the third picture of Fig. 5.5). We note that the same equilibrium can be obtained with any initial conditions.

5.1. Linear filtering of dichotomic Markov noise. The linear filtering of dichotomic Markov noise is a well-known subject in the literature [22,28,35], we have: $A_{1}(x)=-\gamma x+W$, $A_{2}(x)=-\gamma x-W$, with the transition matrix $\left\{q_{i j}\right\}$ given in the example in Introduction, and $\mu_{l}=\lambda,(l=1,2)$ as a parameter of the Poisson statistics. The equilibrium distribution function for Eq. (1.1) is

$$
F_{\text {eq }}(x)=\frac{1}{2}+\frac{\Gamma(b+1 / 2)}{\Gamma(b)} z_{2} F_{1}\left(1 / 2,1-b, 3 / 2, z^{2}\right)
$$

where $b=\lambda /(2 \gamma), z=x \gamma / W,|z| \leqslant 1$, whose density reads as:

$$
p_{\mathrm{eq}}(z)=\frac{\gamma}{W \sqrt{\pi}} \frac{\Gamma(b+1 / 2)}{\Gamma(b)}\left(1-z^{2}\right)^{b-1} .
$$

We solve the Cauchy problem $F_{0 s}(x)=H(x) / 2$ for the evolution equation (1.1) by using the discrete method (2.2) until a time step $N=T / \Delta t$, when we experience the equilibrium, is reached. The definition domain of the equilibrium distribution is $\Omega=[-W / \gamma, W / \gamma]$. Some time dependent numerical plots can be found in [1].

Figure 5.1 shows the global pointwise error (2.7) as a function of $x_{k}$ plotted for the mesh spatial size $N_{x}=81,161,641$. The parameters are $\gamma=0.001$ and $\lambda=0.02$. The convergence is veries as $N_{x}$ increases. The inset shows the equilibrium density (5.2). Figure 5.2 gives the same error computed for the parameters $\gamma=0.05$ and $\lambda=0.02$ and $W=1$. In this case $\hat{E}^{i}$ decreases as $N_{x}$ increases, with the exception of the boundary of the interval.

Fig. 5.1. Global pointwise error for the linear filtering of two-state Markov noise $(\gamma=$ $0.001, \lambda=0.02)$

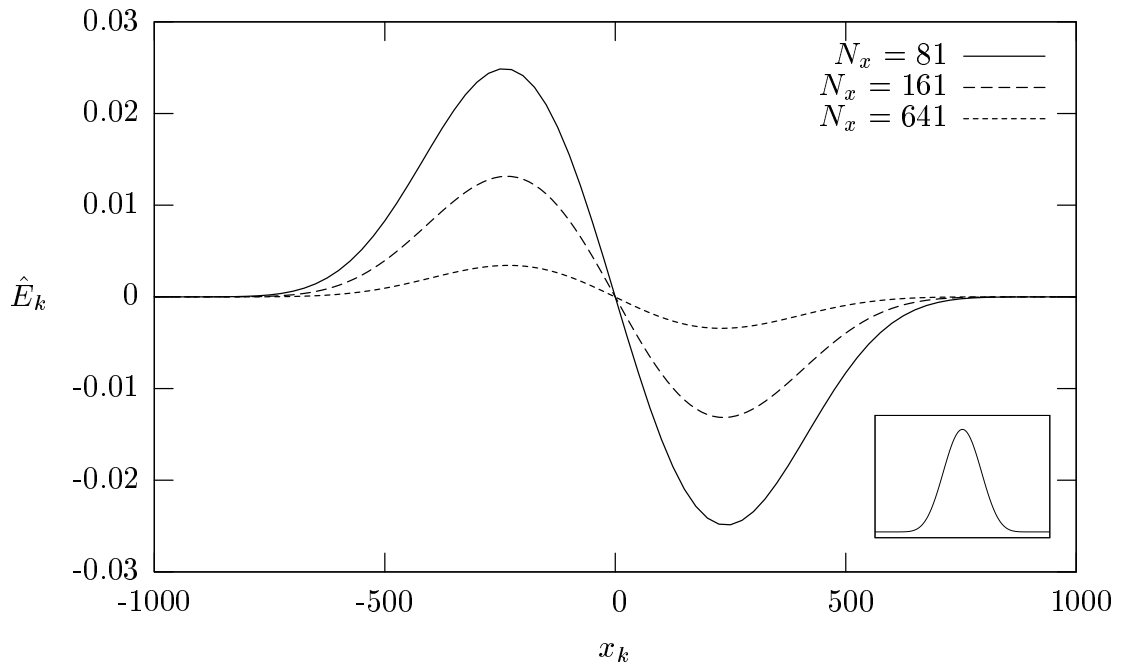


In Fig. 5.3 the global pointwise error is plotted as a function of the size of the spatial mesh. When $\gamma=10^{-3}$, the convergence is linear as predicted by the theory, but for $\gamma=0.05$ convergence is less than linear. This is due to the singularities at the boundary of the domain. If we consider the central fraction 0.9 of the domain, we recover the linear behaviour (see $\gamma=0.05,90 \%)$.

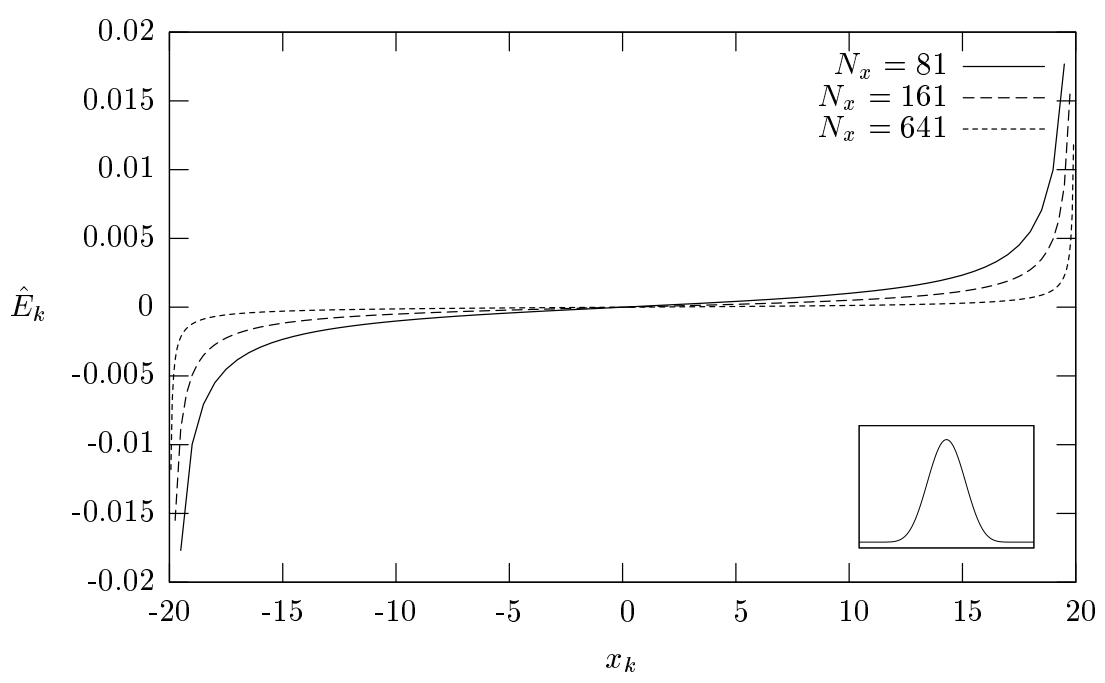

F i g. 5.2. Global pointwise error for the linear filtering of two-state Markov noise $(\gamma=0.05, \lambda=0.02)$

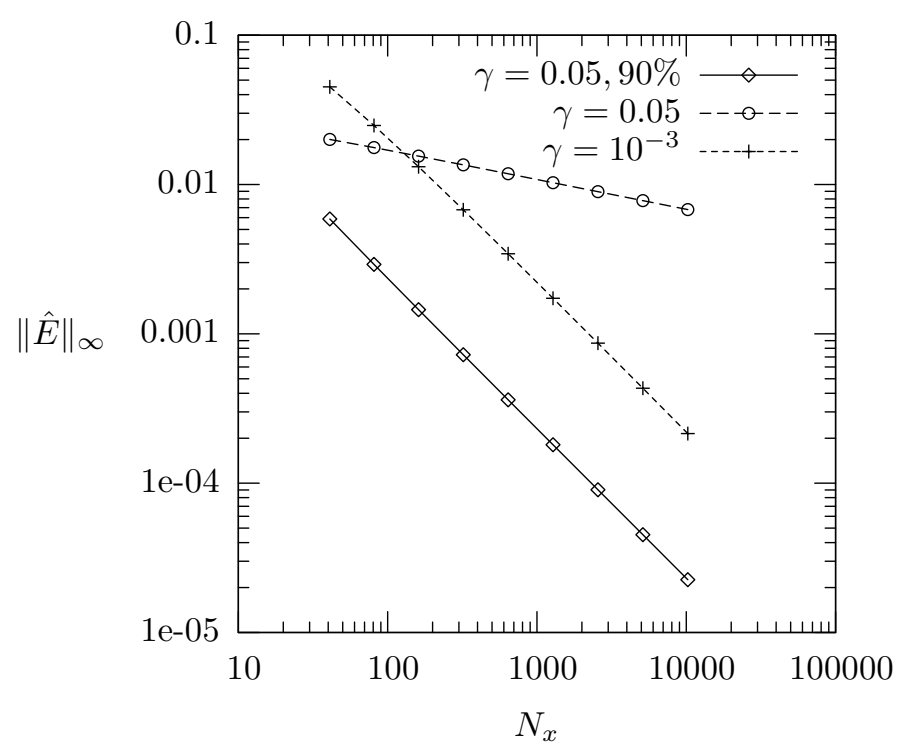

Fig. 5.3. Global pointwise error for the linear filtering of two-state Markov noise versus the mesh size

5.2. Linear filtering of four-state Markov noise. A more complex case is the system of a fluctuation-dissipation process having four relaxation states: $A_{l}(x)=-\gamma_{l} x+W_{l}$ with $l=\{1,2,3,4\}$. This model is solved for the initial condition $F_{0 s}(x)=H(x) / 4$, up to time $T$. The equilibrium domain is given by

$$
x \in \Omega \equiv\left[\min _{s}\left\{\frac{W_{s}}{\gamma_{s}}\right\} ; \max _{s}\left\{\frac{W_{s}}{\gamma_{s}}\right\}\right] .
$$


Figure 5.4 shows the total density distribution function $p\left(x_{k}, T\right)$ when the temporal step $\Delta t$ matches (left) or does not match (right) the CFL condition. The parameters are: $\mu_{s}=4$, $q_{l s}=0.25, \gamma_{s}=10^{-3}, W_{1}=1, W_{2}=-1, W_{3}=2, W_{4}=-2$. By using $\Delta x=4.004$, we get from the CFL condition of Eq. (3.1) that $\Delta t<\Delta t_{\max }=0.250063$. On the left side of Fig. 5.4, we used $\Delta t=0.225056<\Delta t_{\max }$ and $T=545.5$. On the right side $\Delta t=0.5>\Delta t_{\max }$ and the solution is not convergent.

$a$

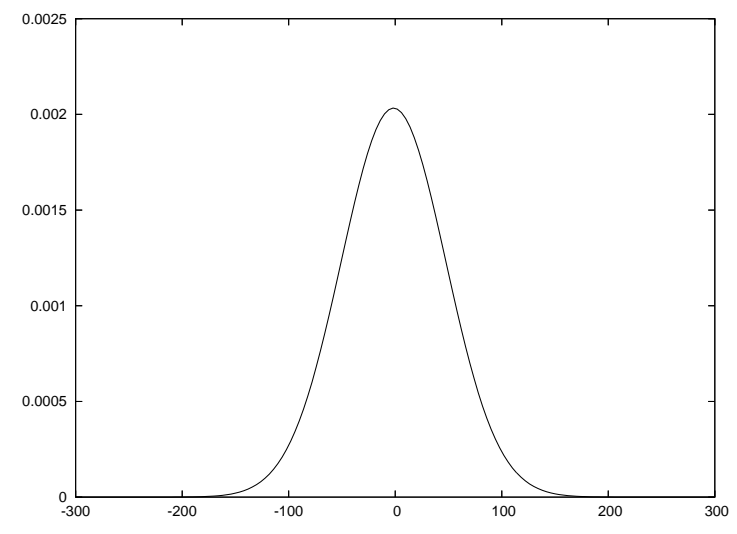

$b$

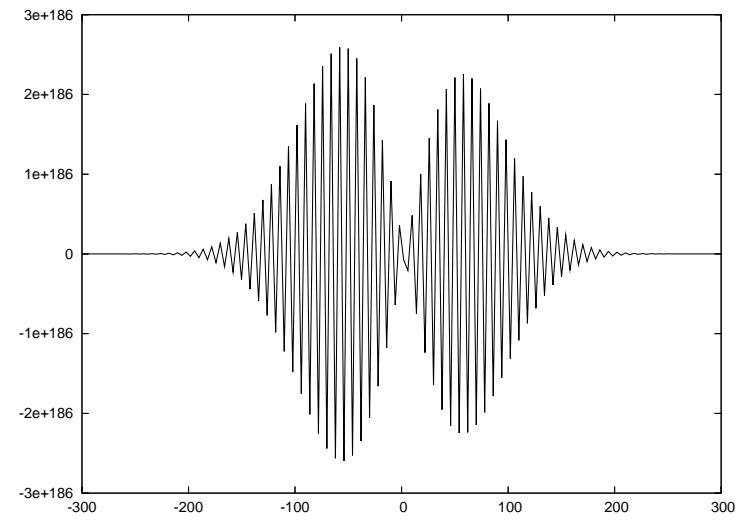

Fig. 5.4. Convergent solution $p\left(x_{k}, T\right)$ of Eq. (1.1), for the linear filtering of four-state Markov noise, when the CFL condition (3.1) is satisfied $(a)$; nonconvergent solution when it is not satisfied $(b)$

In Fig. 5.5 we plot the total probability $p\left(x_{k}, t_{i}\right)$ for fixed times to see how the density distribution evolves. For these plots $\mu_{j}=0.2, \gamma_{j}=0.1$, the other parameters are same as is the previous test. In the same figure we see, only for a qualitative comparison, the histograms obtained by the Monte Carlo simulation $\left(2 \cdot 10^{4}\right.$ samples $)$ of the dynamical equations (1.3),(1.4) and (1.5).

Equilibrium solution. For this problem a reference analytical solution in closed form, such as (5.2) is not available, but we can get it by the Legendre polynomial expansion $F_{l}(x)=\sum_{n} c_{n}^{l} P_{n}(x)$ (see Appendix B). The coefficients $c_{n}^{l}$ are calculated numerically by solving Eqs. (B.1, B.5), with MATLAB. Figure 5.6 shows the global pointwise error as a function of $x$ versus the mesh size $N_{x}$. In Fig. 5.7 the maximum error $\hat{E}$ is plotted versus $N_{x}$, which shows that the linear convergence is confirmed.

5.3. Nonlinear filtering of Markov dichotomous noise. The nonlinear filtering was investigated in [28]; here the driving functions are $A_{1}(x)=-\beta(\sin (x)-1)$ and $A_{2}(x)=$ $-\beta(\sin (x)+1)$, the transition matrix $q_{i j}$ is the same as in Section 5 and the Poisson transition rate is $\lambda$ for both states. For this problem the equilibrium probability density is

$$
p_{e q}(x)=\frac{\sec (x)^{2}}{2 K_{1}(2 \alpha)} e^{-2 \alpha \sec (x)}, \quad|x| \leqslant \frac{\pi}{2}, \quad \alpha=\frac{\lambda}{\beta},
$$

where $K_{1}$ is a modified Bessel function of the second kind.

Figure 5.8 shows the global pointwise error (2.7) with $\alpha=0.1$ for mesh sizes $N_{x}=81$, 161, 641. We see that when $N_{x}$ increases the error decreases. Similar results for $\alpha=0.5$ and $\alpha=4$ are illustrated in Figs. 5.9 and 5.10. 

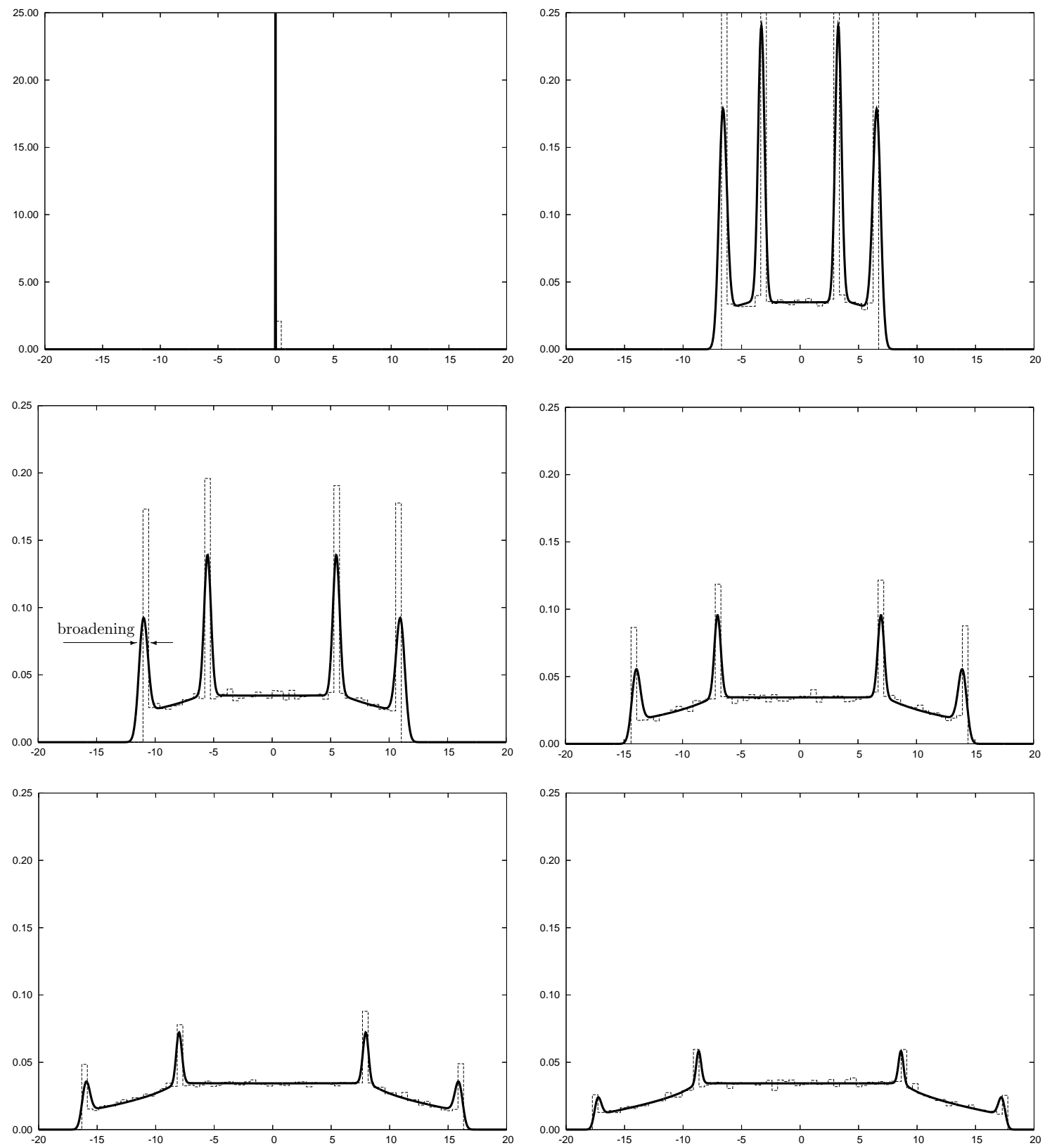

Fig. 5.5. Six snapshots of the temporal evolution of the density probability distribution function $p(x, t)$ for the linear filtering of the four-state noise. $S=4, \Delta t=0.009, \Delta x=0.04004, \mu_{j}=0.2$, $\gamma_{j}=0.1$. From top left to bottom right: $t=\{0,4,8,12,16,20\}$. Histograms from Monte Carlo's simulation are shown by dashed lines 


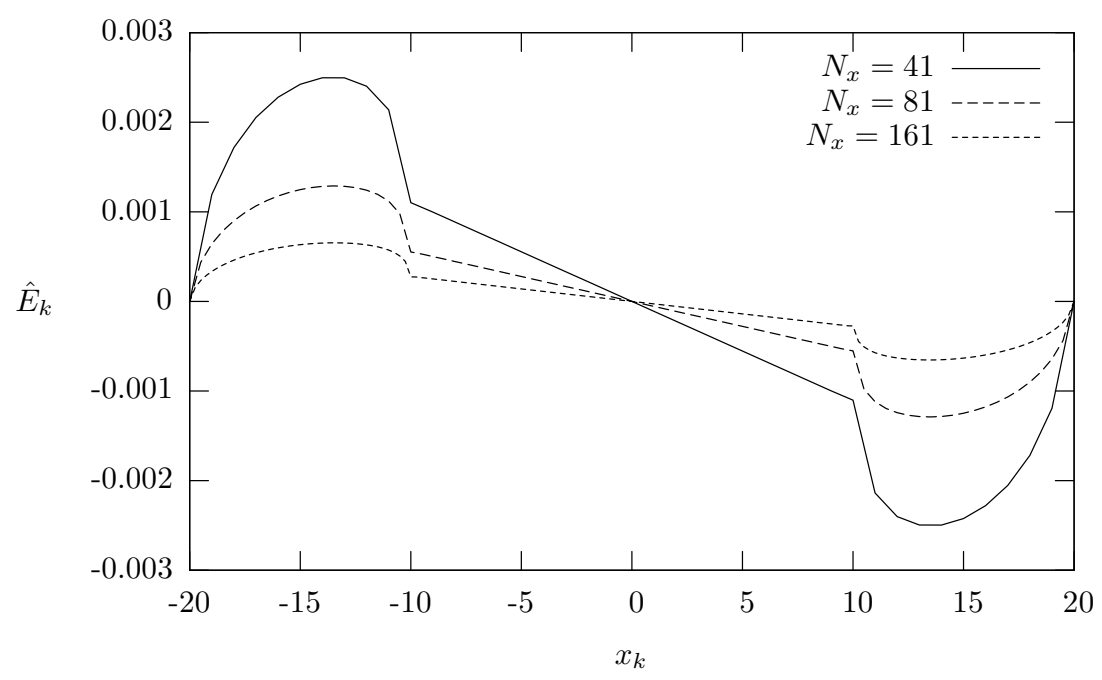

Fig. 5.6. Global pointwise error (2.7) for the four-state noise

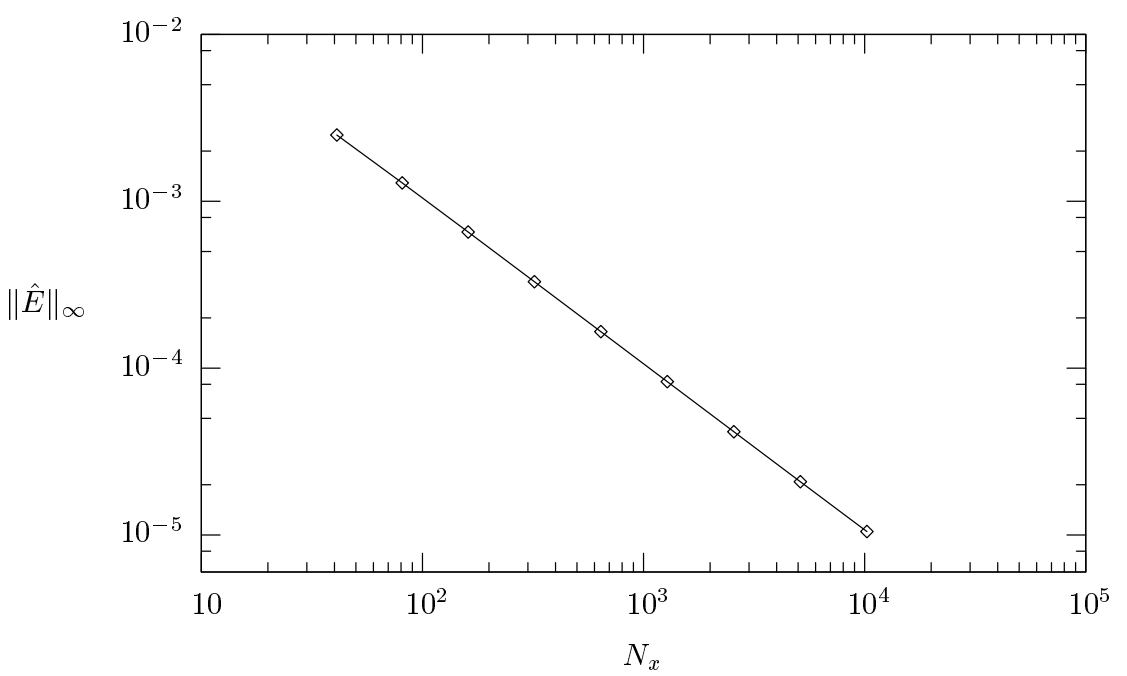

Fig. 5.7. Global error versus the mesh size for the four-state noise

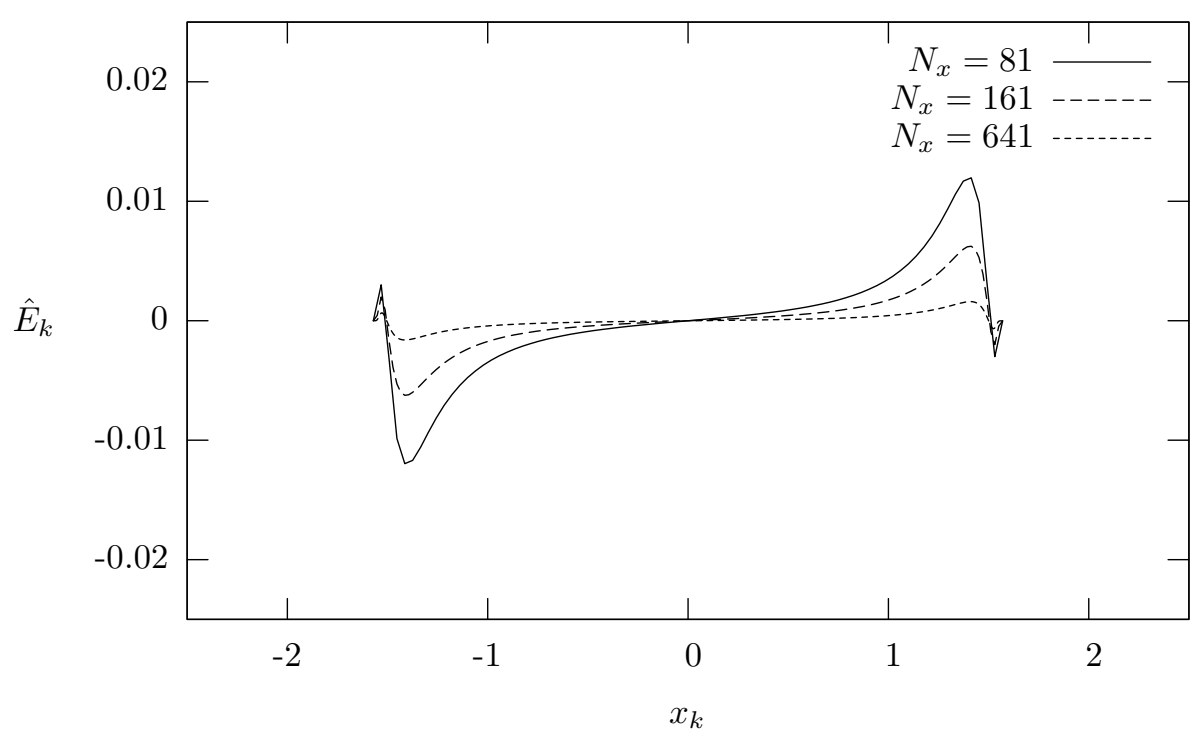

Fig. 5.8. Global pointwise error for the nonlinear filtering, with $\alpha=0.1$ 


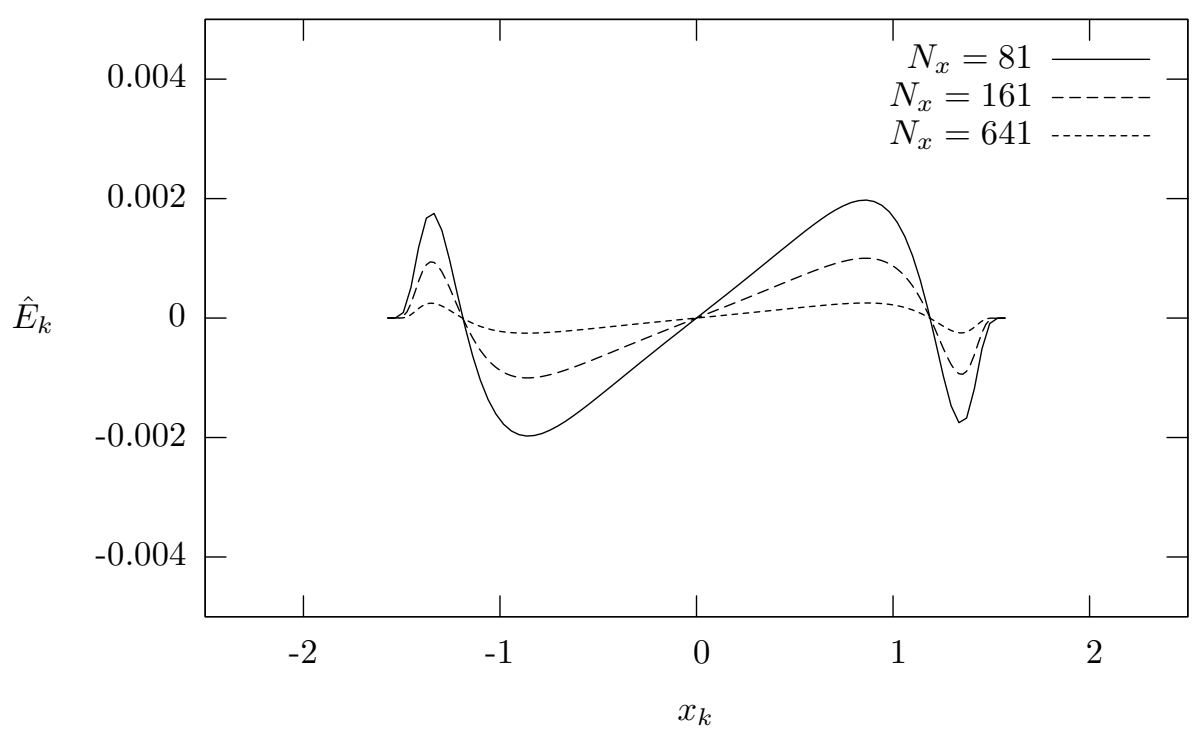

Fig. 5.9. Global pointwise error for the nonlinear filtering, with $\alpha=0.5$

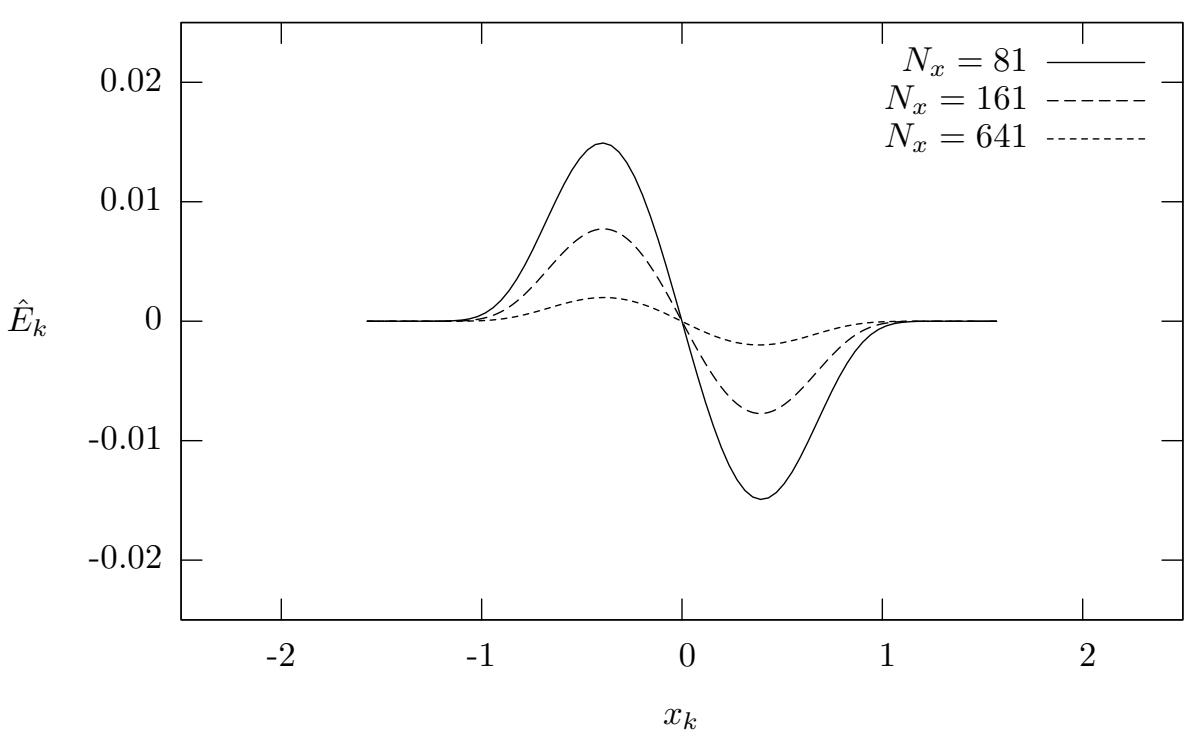

Fig. 5.10. Global pointwise error for the nonlinear filtering, with $\alpha=4$

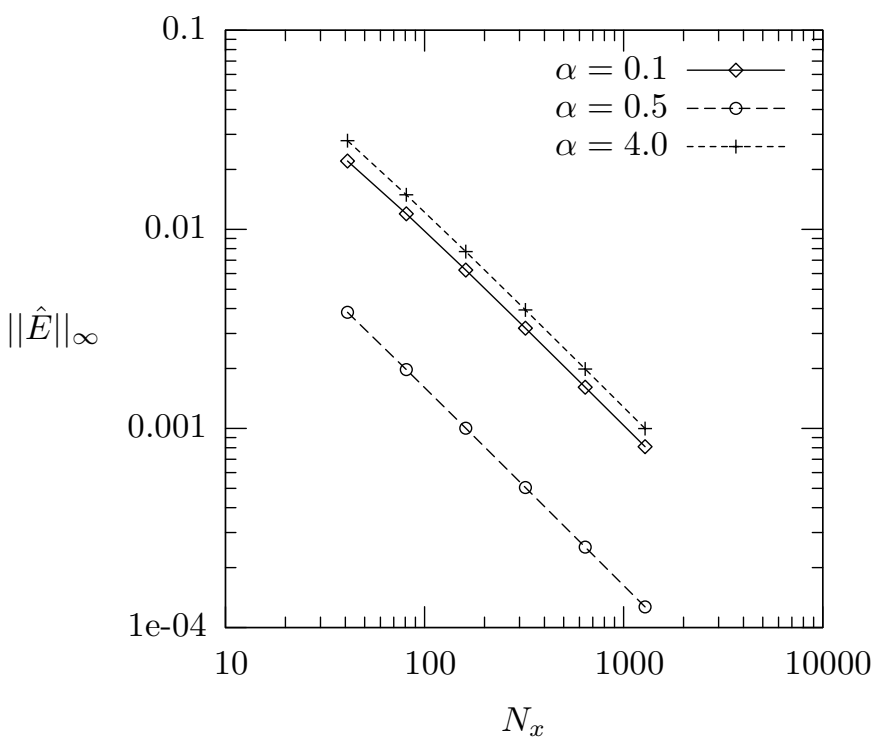

Fig. 5.11. Global pointwise maximum error versus the number of grid points for the nonlinear filter, with $\alpha=0.1,0.5,4.0$ 


\section{Summary and conclusions}

In this paper we have investigated the numerical solution of the Liouville - Master Equation in the form of non-conservative system of hyperbolic equations with balance, for a class of Piecewise Deterministic stochastic processes, obtained by the application of the upwind and explicit Euler scheme. We have shown that the coupling term, originated from a stochastic matrix, does not affect the main features of the scheme, i. e., a CFL-like condition ensures both convergence (Th.3.1) and monotonicity (Th.4.1) of the numerical solution. Moreover, by virtue of the same term jointly with an appropriate norm, we have shown that the global error is bounded by the linear increase with time. As a secondary result, a Legendre series of the equilibrium solution has been found for the case of linear filtering with many states. Some numerical tests have confirmed the theoretical findings.

\section{A. Boundary values for the equilibrium solution}

We consider the equilibrium equation for the distribution function of a Markovian PDP, that comes from Eq. (1.1):

$$
A_{l}(x) \partial_{x} F_{l}(x)=\sum_{s=1}^{S} Q_{l s} F_{s}(x) .
$$

It easy to find a condition for the domain such that the probability densities are vanishing.

Remark A.1. For the regions $x \in \bar{\Omega}$ where all $A_{l}(x)<0$ or $A_{l}(x)>0 \forall l$, the densities $F_{l}^{\prime}(x)$ are vanishing.

In fact, the property $\sum_{l} Q_{l s}=0$ implies that $\sum_{l} A_{l}(x) F_{l}^{\prime}(x)=0$.

Now, let $\Omega \equiv[a, b]$ be a domain for which $F_{l}^{\prime}(x)>0$; the following boundary value problem have to be satisfied:

$$
\begin{gathered}
F_{l}(b)=f_{l}, \\
\sum_{l=1}^{S} f_{l}=1, \\
F_{l}(a)=0 .
\end{gathered}
$$

In order to find solutions of Eq. (A.1) the constants $f_{l}$ have to be determined.

Theorem A.1. Let $[a, b] \subset \mathbb{R}$ be the finite definition domain for the solutions $F_{l}(x)$ of equation (A.1), where (A.2)-(A.4) are satisfied. If the matrix $Q_{l s}$ has rank of one minor of the maximum, then there exists a unique set of boundary values (A.2).

Proof. From the former remark we have that $\sum_{s=1}^{S} Q_{l s} F_{s}(b)=0$, which is an homogenous system of equations. By virtue of the hypothesis there is a row which is a linear combination of the others, let $l^{*}$ be such a row and substitute Eq. (A.3) for it. The new matrix $Q_{l s}^{*}$ has the maximum rank because the inserted row is $(1,1, \ldots, 1)$ and $\sum_{l} Q_{l s}^{*}>0$. Then the system of equations $\sum_{s} Q_{l s}^{*} f_{s}=\delta_{l l^{*}}$ has a unique solution.

Remark A.2. The boundary values (A.2) for the equilibrium equation (A.1) do not depend on $A_{s}(x)$.

Remark A.3. If the stochastic matrix $\left\{q_{i j}\right\}$ is irreducible, then the rank of $Q_{i j}$ is one minor of the maximum. 


\section{B. Expansion of the probability density}

In this section we solve Eq. (A.1) for the linear filtering problem by expanding the densities into the Legendre polynomial series $P_{n}(x): F_{l}^{\prime}(x)=\sum_{n=0}^{\infty} d_{n}^{l} P_{n}(x)$.

Theorem B.1. Let $F_{l}(x), l=1, \ldots, S$, be the solution of $E q .(A .1)$, with $A_{l}(x)=\left(W_{l}-\right.$ $\left.\gamma_{l} x\right)$ and $\max _{l}\left|W_{l} / \gamma_{l}\right|=1$. If $F_{l}^{\prime}(x) \in L^{2}[-1,1]$ such that the Legendre expansion $F_{l}^{\prime}(x)=$ $\sum_{n=0}^{\infty} d_{n}^{l} P_{n}(x)$ exists, then the coefficients $d_{n}^{l}$ satisfy the following equations:

$$
\begin{gathered}
\sum_{s}\left(Q_{l s}-\delta_{l s} \gamma_{s}\right) d_{1}^{s}=-3 W_{l} d_{0}^{l}, \quad \sum_{s}\left(Q_{l s}-2 \delta_{l s} \gamma_{s}\right) d_{2}^{s}=5 \gamma_{l} d_{0}^{l}-5 W_{l} d_{1}^{l} \\
\sum_{s}\left(Q_{l s}-(m+1) \delta_{l s} \gamma_{s}\right) d_{m+1}^{s}=\frac{2 m+3}{2 m-1} \sum_{s}\left(Q_{l s}+m \delta_{l s} \gamma_{s}\right) d_{m-1}^{s}-(2 m+3) W_{l} d_{m}^{l}, \quad m \geqslant 2
\end{gathered}
$$

Proof. We write Eq. (A.1) as

$$
A_{l}(x) F_{l}^{\prime}(x)=\sum_{s=1}^{S} Q_{l s} \int_{-1}^{x} F_{s}^{\prime}(y) d y
$$

As before, for the case of the relaxation processes we get

$$
\begin{gathered}
\sum_{n=0}^{\infty} \int_{-1}^{1} d_{n}^{l} P_{m}(x)\left(W_{l}-\gamma_{l} x\right) P_{n}(x) d x= \\
\sum_{s=1}^{S} Q_{l s}\left(d_{0}^{s}\left\langle P_{m}, P_{0}+P_{1}\right\rangle+\sum_{n=1}^{\infty} \frac{d_{n}^{s}}{2 n+1}\left(\left\langle P_{m}, P_{n+1}\right\rangle-\left\langle P_{m}, P_{n-1}\right\rangle\right)\right),
\end{gathered}
$$

where we have used the relation

$$
\int_{-1}^{x} P_{n}(y) d y=\frac{P_{n+1}(x)-P_{n-1}(x)}{2 n+1}, \quad n \geqslant 1,
$$

and $\left\langle P_{m}, P_{n}\right\rangle=\int_{-1}^{1} P_{m}(x) P_{n}(x) d x$.

By developing the integrals

$$
d_{m}^{l} W_{l}-\frac{\gamma_{l}(2 m+1)}{2} \sum_{n} d_{n}^{l}\left\langle P_{m}, x P_{n}\right\rangle=\sum_{s} Q_{l s}\left(d_{0}^{s}\left(\delta_{m 0}+\delta_{m 1}\right)+\frac{d_{m-1}^{s}}{2 m-1}-\frac{d_{m+1}^{s}}{2 m+3}\right) .
$$

The integral on the left hand side is

$$
\frac{2(m+1) \delta_{m+1, n}}{(2 m+1)(2 m+3)}+\frac{2 m \delta_{m-1, n}}{(2 m-1)(2 m+1)}
$$

and provided that $\sum_{s} Q_{l s} d_{0}^{s}=\sum_{s} Q_{l s} F_{s}(1) / 2=0$, we get the thesis.

The distribution functions are calculated by the Legendre expansion: $F_{l}(x)=\sum_{n=0}^{\infty} c_{n}^{l} \times$ $P_{n}(x)$, where $c_{n}^{l}$ are related to $d_{n}^{l}$ by

$$
c_{0}^{l}=d_{0}^{l}-d_{1}^{l} / 3, \quad c_{n}^{l}=\frac{d_{n-1}^{l}}{2 n-1}-\frac{d_{n+1}^{l}}{2 n+3}, \quad \text { if } n>0 .
$$

We note that Eq. (A.1) can be scaled in a such way that $\max _{l}\left|W_{l} / \gamma_{l}\right|=1$.

Finally, we note that if $F^{\prime}(x) \in L^{2}[a, b]$, then the Legendre expansion of $F(x)$ is uniformly convergent (see, e.g., $[29,32]$ ). This property justifies the numerical test of Sect. 5 . 


\section{References}

1. M. Annunziato, Non-gaussian equilibrium distribution arising from the Langevin equation, Phys. Rev. E, 65 (2002), pp. 21113-1-6.

2. M. Annunziato, A finite difference method for piecewise deterministic processes with memory, Math. Mod. and Analysis, 12 (2007), pp. 157-178.

3. M. Bologna, P. Grigolini, and B. J. West, Strange kinetics: conflict between density and trajectory description, Chem. Phys. 284 (2002), pp. 115-128.

4. A. Budini, M. O. Cáceres, Functional characterization of generalized Langevin equations, J. Phys. A: Math. Gen. 37 (2004), pp. 5959-5981.

5. M. O. Cáceres, Computing a non-Maxwellian velocity distribution from first principles, Phys. Rev. E, 67 (2003), pp. $016102-1-5$.

6. M. Castro, J. Macías, and C. Parés, A Q-scheme for a class of systems of coupled conservation laws with source term. Application to a two-layer 1-D shallow water system, Math. Mod. Num. Anal. 35 (2001), pp. $107-127$.

7. O. L. V. Costa, Stationary distributions for piecewise-deterministic Markov processes, J. Appl. Prob. 27 (1990), pp. 60-73.

8. M. H. A. Davis, Markov Models and Optimisation, Monograph on Statistics and Applied Probability 49, Chapman \& Hall/CRC, 1993.

9. M. H. A. Davis, Piecewise-Deterministic Markov Processes: A General Class of Non-Diffusion Stochastic Models, J. of the Royal Stat. Soc., Series B, 46 (1984), pp. 353-388.

10. A. Di Crescenzo, On random motions with velocities alternating at Erlang-Distributed random times, Adv. Appl. Prob. 33 (2001), pp. 690-701.

11. A. Di Crescenzo and B. Martinucci, Random motion with gamma-distributed alternating velocities in biological modeling, Computer Aided Systems Theory EUROCAST 2007, Lecture Notes in Computer Science, Springer Verlag, pp. 163-170.

12. F. Dufour and O. L. V. Costa, Stability of piecewise-deterministic Markov processes, SIAM J. Control Optim. 37 (1999), pp. 1483-1502.

13. R. Filliger and M.O. Hongler, Supersymmetry in random two-velocity processes, Physica A, 332 (2004), pp. 141-150.

14. C.W. Gardiner, Handbook of Stochastic Methods, Springer-Verlag, Berlin — Heidelberg — New York, 2nd Ed., 1985.

15. W. Horsthemke, Spatial instabilities in reaction random walks with direction-independent kinetics, Phys. Rev. E, 60 (1999), pp. 2651-2663.

16. W. Hundsdorfer and J. G. Verwer, Numerical solution of Time-Dependent Adverction-Diffusion-Reaction Equations, Springer Series in Computational Mathematics, Vol. 33, Springer-Verlag, 2003.

17. E. Jakeman and E. Renshaw, Correlated random-walk model for scattering, J. Opt. Soc. Am. A, 4 (1987), pp. 1206-1212.

18. E. Jakeman and K. D. Ridley, The statistics of a filtered telegraph signal, J. Phys. A, 32 (1999), pp. 8803-8821.

19. V. Jovanović and C. Rohde, Error estimate for finite volume approximations of classical solutions for nonlinear systems of hyperbolic balance laws, SIAM J. Numer. Anal. 43 (2006), pp. 2423-2449.

20. K. Kitahara et al., Phase Diagrams of Noise Induced Transitions, Prog. Theor. Phys. 64 (1980), pp. 1233-1247.

21. J. Masoliver and J. M. Porrà, Harmonic oscillators driven by colored noise: Crossovers, resonances, and spectra, Phys. Rev. E. 48 (1993), pp. 4309-4319.

22. G. N. Milstein and Yu. M. Repin, Action of Markov process on a system of differential equations, Differential Equations 5 (1972), pp. 1010-1019 (Translated from: Differentsial Uravneniya 5 (1969), pp. 13711384).

23. A. Morita, Free Brownian motion of a particle driven by a dichotomous random force, Phys. Rev. A. 41 (1990), pp. $754-760$.

24. K. W. Morton and D. F. Mayers, Numerical Solution of Partial Differential Equations, Cambridge University Press, 1994.

25. M. L. Muñoz-Ruiz and C. Parés, Godunov method for nonconservative hyperbolic systems, M2AN, 41 (2007), pp. 169-185.

26. H. G. Othmer, S. R. Dunbar, and W. Alt, Models of dispersal in biological systems, J. Math. Biol. 26 (1988), pp. 263-298. 
27. R. F. Pawula and O. Rice, On Filtered Binary Processes, IEEE Trans. Inf. Th. Vol. IT-32, (1986), pp. $63-72$.

28. R.F.Pawula, The probability density and level-crossings of first-order non-linear systems driven by the random telegraph signal, Int. J. Control. 25 (1977), pp. 283-292.

29. J.Prasad and H. Hayashy, On the uniform convergence of Fourier - Jacobi series, SIAM J. of Numer. Anal. 10 (1973), pp. 23-27.

30. R. J. LeVeque, Numerical Methods for conservation Laws, Birkhäuser, 1992.

31. V. Starikovičius, R. Čiegis and A. Jakušev, Analysis of upwind and high-resolution schemes for solving convection dominated problems in porous media, Math. Mod. and Analysis. 11 (2006), pp. 451-474.

32. P.K. Suetin, On the representation of continuous and differentiable functions by Fourier series of Legendre polynomials, Dokl. Acad. Nauk SSSR. 158 (1964), pp.1275-1277 (Engl. Transl. in Soviet Math. Dokl. 5 (1964), pp. 1408).

33. C. Van den Broeck and P. Hänggi, Activation rates for nonlinear stochastic flows driven by nonGaussian noise, Phys. Rev. A. 30 (1984), pp. 2730-2736.

34. B. van Leer, Upwind and high resolution methods for compressible flow: from donor cell to residualdistribution schemes, Comm. Comp. Phys. 1 (2006), pp. 192-206.

35. W. M. Wonham, J. Electron. Control 6 (1959), pp. 376-383. 